\title{
Organizing pneumonia: a rare pulmonary manifestation of well- controlled ulcerative colitis
}

\author{
Xiaoming Zhou, Yu Chen, Li Zhao \\ Department of Respiratory Medicine, Shengjing Hospital, China Medical University, Shenyang 110004, China \\ Correspondence to: Xiaoming Zhou. Sanhao Street 36\#, Heping District, Shenyang 110004, China. Email: zhouxmcmu@163.com.
}

Submitted Apr 30, 2018. Accepted for publication Jul 19, 2018.

doi: $10.21037 /$ jtd.2018.07.102

View this article at: http://dx.doi.org/10.21037/jtd.2018.07.102

\section{Introduction}

Inflammatory bowel disease (IBD) is thought of as a multiorgan disease with frequent extraintestinal manifestations. Pulmonary manifestations of IBD are rare, but when they occur, they pose a challenge to definitive diagnosis. Studies have documented the occurrence of airway diseases, interstitial lung disease, organizing pneumonia $(\mathrm{OP})$, pulmonary nodules, serositis and druginduced lung disease (1-3). However, if pulmonary involvement occurs while IBD is under control, it is far more difficult to diagnose, primarily because the clinical manifestations are complicated by the effects of chemotherapy, concurrent infections and other factors. The failure to definitively diagnose pulmonary involvement then makes further treatment difficult, jeopardizing the patient's prognosis.

\section{Case presentation}

A 62-year-old male with ulcerative colitis was admitted to hospital with fever. The initial diagnosis of ulcerative colitis had been made 2 years earlier on the basis of bloody, purulent stool. At the time, the patient was given oral prednisolone therapy with the initial dosage of oral prednisone of $0.5 \mathrm{mg} / \mathrm{kg}$, tapered gradually for 4 months, and then he was started on low-dose mesalazine (Etiasa, 1.5 g/day; Ethypharm, France), which the patient had been taking for 1 year by the time of admission without further corticosteroid treatment. Four months before admission, the bloody mucous stool stopped. For 7 days before admission, the patient experienced fever with a peak temperature of $39^{\circ} \mathrm{C}$ but no chills or obvious respiratory symptoms. The patient reported no prior history of pulmonary symptoms or lung disease, no history of cigarette smoke, no occupational exposure, no history of extraintestinal manifestations of IBD and no recent travel.

Physical examination revealed the stable vital signs (heart rate $85 \mathrm{bpm}$; blood pressure $120 / 60 \mathrm{mmHg}$; respiration rate 18 breaths per minute). He had reduced breath sounds in the right lung without wheezing or rubs.

Laboratory data included a white blood cell count of $8.1 \times 10^{9} / \mathrm{L}$ (normal, $4 \times 10^{9}-10 \times 10^{9} / \mathrm{L}$ ), with the following differential: $57.1 \%$ neutrophils, $30.4 \%$ lymphocytes and $0.5 \%$ eosinophils. Microcytic hypochromic anemia was present (hemoglobin $92 \mathrm{~g} / \mathrm{L}$; mean cell volume $67 \mathrm{fL}$; mean corpuscular hemoglobin concentration $303 \mathrm{~g} / \mathrm{L}$ ), and platelet count was $339 \times 10^{9} / \mathrm{L}$. Arterial blood gas analysis showed $\mathrm{PaO}_{2} 81 \mathrm{mmHg}, \mathrm{PaCO}_{2} 37 \mathrm{mmHg}, \mathrm{pH} 7.45$, and oxygen saturation $96 \%$ (at the time of admission on room air). Erythrocyte sedimentation rate was $98 \mathrm{~mm} / \mathrm{h}$. Computed tomography (CT) revealed consolidation of the right middle and lower lobes with ipsilateral pleural effusion (Figure 1). Colonoscopy showed that the ulcerative colitis was well-controlled compared to the analysis two years prior.

These analyses on admission led to a preliminary diagnosis of community-acquired pneumonia with parapneumonic pleural effusion. The patient was administered piperacillin/tazobactam ( $4.5 \mathrm{~g}$ ) intravenously every 8 hours. After 2 days, the patient was afebrile. Antibiotic therapy lasted for 10 days. At the end of antibiotic therapy, chest CT showed partial resolution of the lesion in the right lung but new involvement of the left lung, presented as patchy, ground-glass opacities and scattered cysts (Figure 1C,D). Bronchoscopy did not find abnormality, and analysis of bronchoalveolar lavage fluid (BALF) showed 

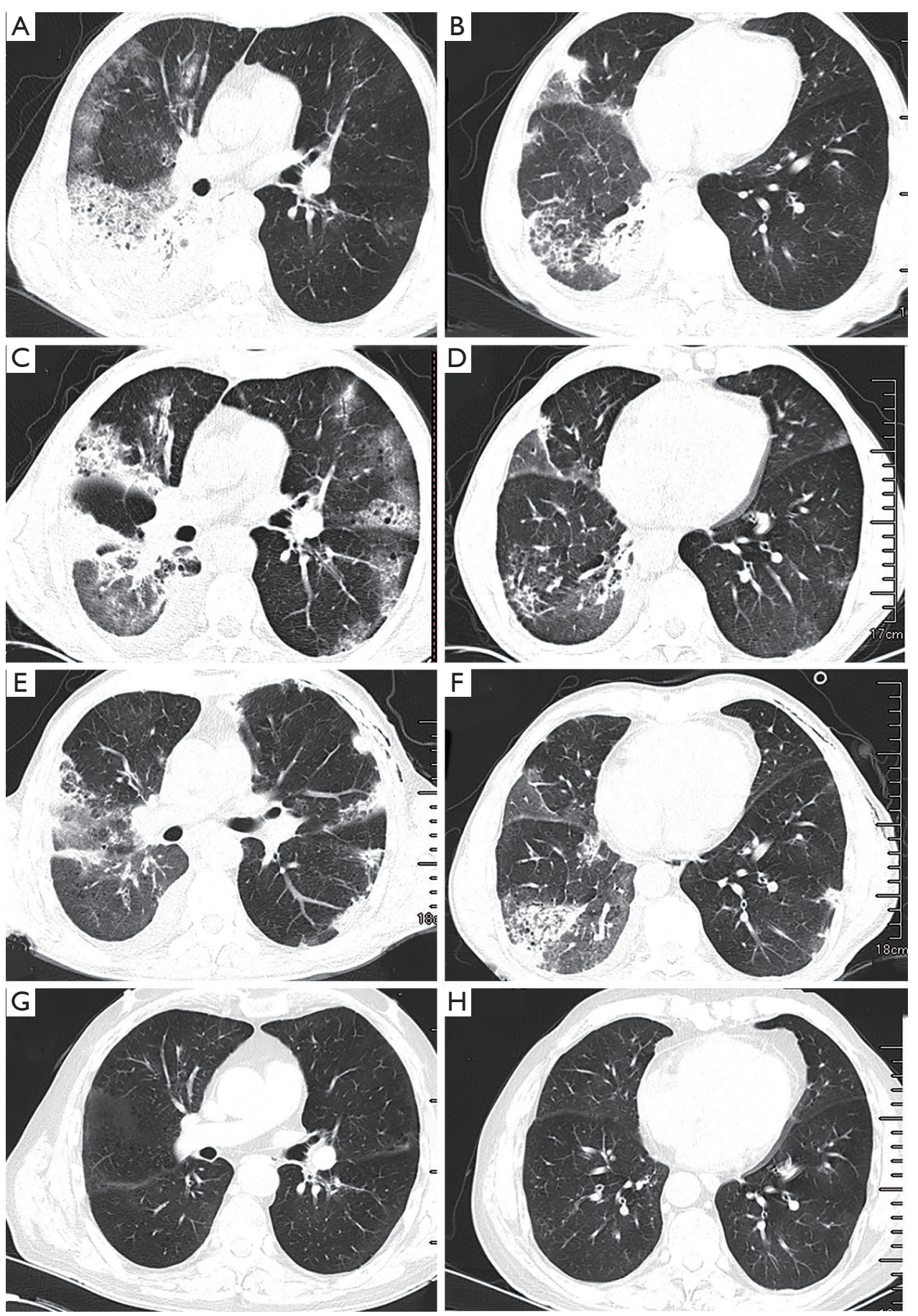

Figure 1 Sequential chest CT scan of the patient from admission to the resolution of the disease. (A,B) Chest CT of the patient on admission which presented as consolidation of the right middle and lower lobes with isolateral pleural effusion; (C,D) chest CT of the patient with 10-day antibiotic therapy which presented as partial absorption of the right lung but involvement of the left lung, which presented as patchy, ground-glass opacity and scattered cysts; $(\mathrm{E}, \mathrm{F})$ chest $\mathrm{CT}$ of the patient 24 days after the admission which presented as no obvious absorption, compared with the chest $\mathrm{CT}$ of 2 weeks ago; $(\mathrm{G}, \mathrm{H})$ chest $\mathrm{CT}$ of the patient after three-month prednisolone therapy: obvious absorption of the bilateral patchy, ground-glass opacities. CT, computed tomography. 


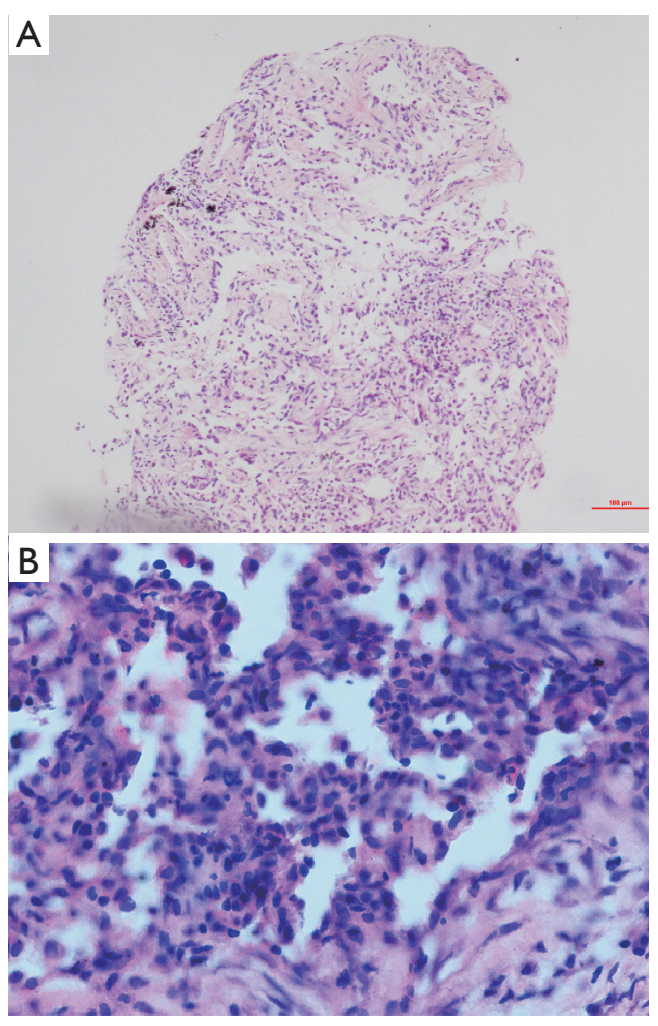

Figure 2 Pathology of the lung biopsy. (A) The pathological examination of the percutaneous lung biopsy based on hematoxylin and eosin-staining $(\times 100)$; (B) the pathological examination of the percutaneous lung biopsy based on hematoxylin and eosin-staining ( $\times 400)$ : cellular and lymphocytic alveolitis, non-specific alveolar reaction, and the IAFD in the alveolar space associated with organizing pneumonia. IAFD, intra-alveolar fibrin deposition.

$21 \%$ lymphocytes, $12 \%$ neutrophils, and $67 \%$ macrophages, without eosinophils or detectable microorganisms. The ratio of $\mathrm{CD}^{+}$to $\mathrm{CD}^{+}$lymphocytes in BALF was 0.61 . BALF was sent for the detection of bacteria, fungi, tuberculosis and PCP without positive results. Antinuclear antibodies in serum were negative. Transbronchial lung biopsy produced four samples (lower lobe of right lung) revealing atelectatic alveolar tissue with scattered foamy cells as well as chronic inflammatory cell infiltration and congestion. Pulmonary function tests revealed reduced diffusion function (DLCO\%, 70\%) with mildly reduced ventilatory function (vital capacity \% predictive value, $75 \%$ ). Percutaneous lung biopsy and corticosteroid therapy were proposed but the patient refused further invasive examination and therapy. Therefore, the patient was discharged and observed closely.
Two weeks later, the patient reported no discomfort, but the axial sections of chest CT showed no obvious resolution (Figure $1 E, F$ ). In order to get definite diagnosis, the patient was hospitalized again for percutaneous lung biopsy (four samples from the lower lobe of right lung) and further therapy. The biopsy showed lymphocytic alveolitis, nonspecific alveolar reaction associated with $\mathrm{OP}$ (Figure 2), for which a side-effect of mesalazine could not be excluded due to the rare evidence of active IBD and other extraintestinal manifestations. Therefore, mesalazine was discontinued and the patient was tested by negative interferon-gamma release assay (IGRA) to exclude latent tuberculosis. One month later, the patient still presented recurrent dry cough with no fever, therefore prednisolone was given to the patient for 1 month at a dose of $48 \mathrm{mg} / \mathrm{d}$. Chest CT at the end of that month showed obvious resolution of the bilateral infiltrations (Figure 1G,H). Prednisolone therapy was tapered by $4 \mathrm{mg} /$ week. Over the entire course of glucocorticoid treatment, the ulcerative colitis remained stable with no stool changes or other complications. Due to the good gastrointestinal response of ulcerative colitis to mesalazine, the patient insisted on restarting mesalazine after the complete resolution of OP on the CT scan at the same dose as before OP occurrence. The patient took mesalazine half a year later with no occurrence of similar pulmonary complication during the 2-year follow-up period.

\section{Discussion}

Respiratory manifestations of ulcerative colitis are rarely seen, but they can present in diverse forms (1-4) and can appear before IBD is diagnosed or weeks even years afterwards. This makes diagnosing IBD-associated lung disease challenging. In fact, respiratory involvement of IBD can be asymptomatic and detectable only by pulmonary function tests or high-resolution CT (5). Evidence suggests that more than $50 \%$ of patients with IBD show impaired pulmonary function without clinical or radiographic findings (5). With good control of IBD, active pulmonary complication could also occur without other system involvement, even without drug-induced pulmonary adverse effects (6).

In the present case, the patient did not show any respiratory symptoms on admission or afterwards, but the CT scan showed bilateral infiltration of the lungs and progression of lung involvement. Pathological examination clearly showed OP of the lung. 
One unanswered question about our patient is whether the respiratory involvement was an extraintestinal manifestation of ulcerative colitis or an adverse effect of mesalazine therapy. Previous studies suggested that the most frequent pathological findings for pulmonary changes related to ulcerative colitis are interstitial lymphocyte infiltration, alveolar fibrinous exudates, progressive fibrosis and OP. OP may be associated with IBD progression (3,7-11). According to one literature review, OP has been described only in $2 \%(9 / 400)$ and $13 \%(21 / 155)$ of patients with pulmonary involvement in IBD, more commonly in UC (11). When the patient presented with fever, we should be aware of the respiratory complications.

There have been scattered case reports of the association of amino salicylate mesalazine and OP (12-14). In spite of the potential pulmonary toxicity of mesalazine, a definite causal relationship between mesalazine and OP has not been made in either case reports or animal experiments. Nevertheless, these changes may also be the result of druginduced pulmonary toxicity, highlighting the limitations of BALF analysis and pathology for understanding the cause of pulmonary manifestations. This is an important question because the answer determines the most appropriate therapy. If the pulmonary complications are an extraintestinal manifestation of IBD, the dose of mesalazine or sulfasalazine may need to be increased. If, however, the pulmonary complications are due to drug-induced toxicity, then a dose increase may be unwise. Clinicians should take several factors into account when making a "best guess" regarding the cause of the pulmonary complications. The history, activity and clinical presentation of a patient's IBD, especially any extraintestinal manifestations, may suggest that pulmonary complications are related to IBD. Conversely, rapid improvement of pulmonary signs and symptoms upon withdrawal of the drug suggests that the complications are drug-induced. However, discontinuing a drug may not rapidly cause radiological improvement, even if the drug is causing the pulmonary complications, and waiting to observe a clinical response may delay appropriate treatment. Thus, pathological examination may be the most effective method for narrowing the differential diagnosis and providing a definitive diagnosis. However, for complicated conditions such as in our case, the causes of OP remain confusing. What is interesting regarding the cause of the OP is that the reusage of the mesalazine did not induce a similar response of lung during 2-year follow-up.

This case report highlights the possibility that latent pulmonary involvement in ulcerative colitis can arise not only from the colitis itself but also as an adverse effect of the drugs used to treat it, usually sulfasalazine or mesalazine, that are quite difficult to differentiate. Pulmonary involvement in IBD patients who do not have active IBD or other extraintestinal manifestations may indicate drug-induced lung disease. However, the pulmonary complications could occur without a flare of ulcerative colitis. Corticosteroid treatment based on definitive pathological diagnosis may help to confirm the diagnosis and initiate proper treatment in a timely fashion, thus improving recovery from pulmonary involvement of ulcerative colitis.

\section{Acknowledgements}

None.

\section{Footnote}

Conflicts of Interest: The authors have no conflicts of interest to declare.

Informed Consent: Written informed consent was obtained from the patient for publication of this manuscript and any accompanying images.

\section{References}

1. Eade OE, Smith CL, Alexander JR, et al. Pulmonary function in patients with inflammatory bowel disease. Am J Gastroenterol 1980;73:154-6.

2. Higenbottam T, Cochrane GM, Clark TJ, et al. Bronchial disease in ulcerative colitis. Thorax 1980;35:581-5.

3. Williams T, Eidus L, Thomas P. Fibrosing alveolitis, bronchiolitis obliterans, and sulfasalazine therapy. Chest 1982;81:766-8.

4. Cohen M, Sahn SA. Bronchiectasis in systemic diseases. Chest 1999;116:1063-74.

5. Kuzela L, Vavrecka A, Prikazska M, et al. Pulmonary complications in patients with inflammatory bowel disease. Hepatogastroenterology 1999;46:1714-9.

6. Ikehara N, Fujimoto K, Sadohara J, et al. Interstitial Pneumonia associated with ulcerative colitis: highresolution computed tomography and pathologic findings. J Thorac Imaging 2013;28:W21-3.

7. Abraham A, Karakurum A. Acute respiratory failure secondary to mesalamine-induced interstitial pneumonitis. BMJ Case Rep 2013;2013. doi: 10.1136/bcr-2013-009834. 
8. Nagy F, Molnar T, Makula E, et al. A case of interstitial pneumonitis in a patient with ulcerative colitis treated with azathioprine. World J Gastroenterol 2007;13:316-9.

9. Kevans D, Greene J, Galvin L, et al. Mesalazine-induced bronchiolitis obliterans organizing pneumonia (BOOP) in a patient with ulcerative colitis and primary sclerosing cholangitis. Inflamm Bowel Dis 2011;17:E137-8.

10. Aydoğdu M, Gürsel G, Özyilmaz E, et al. A case of ulcerative colitis complicated with bronchiolitis obliterans organizing pneumonia (BOOP) and air leak syndrome. Turk J Gastroenterol 2012;23:590-5.

11. Jang EC, Choi SJ, Cho JH, et al. Organizing pneumonia

Cite this article as: Zhou X, Chen Y, Zhao L. Organizing pneumonia: a rare pulmonary manifestation of well-controlled ulcerative colitis. J Thorac Dis 2018;10(8):E634-E638. doi: 10.21037/jtd.2018.07.102 presenting after ulcerative colitis remission. J Thorac Dis 2013;5:E71-3.

12. Costa JM, Carvalho SD, Soares JB. Mesalazine-induced Bronchiolitis Obliterans with Organizing Pneumonia in a young patient with Ulcerative Colitis. J Crohns Colitis 2018. [Epub ahead of print].

13. Akiyama N, Yokomura K, Nozue T, et al. Three cases of drug-induced pneumonia caused by mesalazine. Arerugi 2015;64:1334-40.

14. Michy B, Raymond S, Graffin B. Organizing pneumonia during treatment with mesalazine. Rev Mal Respir 2014;31:70-7. 